\title{
¿Es la Fibromialgia un trastorno depresivo con síntomas somáticos o es un síndrome de sensibilización central con comorbilidad depresiva?
}

\author{
Díaz Robredo LA. ${ }^{1}$, Robles Sánchez JI. ${ }^{2}$
}

Sanid. mil. 2014; 70 (2): 76-87; ISSN: 1887-8571

\begin{abstract}
RESUMEN
Introducción: El objetivo de este estudio es evaluar los factores de personalidad, calidad de vida y hormonales de pacientes diagnosticados de Fibromialgia para comparar esta enfermedad con otras definidas como Síndromes de Sensibilización Central. Método: Para ello se emplearon el Personality Assessment Inventory, el Cuestionario de Impacto de Fibromialgia y muestras de cortisol salivar en un conjunto experimental de 48 personas. Resultados: Los resultados obtenidos señalan que los afectados por esta enfermedad poseen unas características de personalidad muy determinadas que no les asemejan ni con una situación de enfermedad mental psiquiátrica ni tampoco con niveles de normalidad absoluta, predominando puntuaciones altas en ansiedad, depresión y quejas somáticas, pero normales en otras como Manía, Paranoidismo o Esquizofrenia. Este patrón psicológico los hace más sensibles a los estímulos y mecanismos relacionados con el estrés. Los análisis exploratorios y confirmatorios realizados revelan que los patrones de personalidad formados por las variables psicológicas Ansiedad, Somatización y Depresión son las que más peso tienen en la calidad de vida de los pacientes y que en cambio la variable cortisol no tiene apenas influencia. Conclusiones: Las conclusiones extraídas por este estudio aconsejan buscar tratamientos terapéuticos que tengan en cuenta estas variables de personalidad señaladas, evitando su confusión con enfermedades psiquiátricas.
\end{abstract}

PALABRAS CLAVE: Síndrome de Sensibilización Central, Fibromialgia, Depresión, Ansiedad, Estrés, Personalidad.

¿Is Fibromyalgia a depressive disorder with somatics symptoms or a central sensitization syndrome with depressive comorbidity? SUMMARY: Introduction: The aim of this work is to evaluate personality factors, quality of life and hormone levels in patients with Fibromyalgia in order to compare this disease with others Central Sensitivity Syndromes. Method: 48 subjects were measured by Personal Assessment Inventory, Fibromyalgia Impact Questionnaire and salivary cortisol. Results: The results show us that people affected by this disease have a very determined personality different to a mental disease and at the same time different to healthy people, predominating high scores in anxiety, depression and physical complains, but obtaining normal scores in other scales such as Mania, Paranoia or Schizofrenia. This psychological pattern makes them more sensitive to signals and mechanisms related to stress. Exploratory and confirmatory analysis reveal that personality patterns made up of Anxiety, Somatization and Depression are the most important in the quality of life of this patients whereas cortisol has very little effect on it. Conclusions: The conclusions extracted from this work lead to look for therapeutic treatments which take into account this mentioned personality variables, trying to avoid any confusion with other mental diseases.

KEY WORDS: Central Sensitization Syndrome, Fibromyalgia, Depression, Anxiety, Stress, Personality.

\section{INTRODUCCIÓN}

La Fibromialgia, por su sintomatología, ha sido relacionada con un grupo de problemas de salud que a día de hoy siguen sin contar con una explicación médica clara. Conceptos como enfermedades psicosomáticas, síndromes somáticos funcionales o síndrome de sensibilización central, tal y como se les denomina actualmente, sirven habitualmente para agrupar enfermedades que abarcan varios sistemas corporales: la fibromialgia (FM en adelante); el síndrome de intestino irritable o la dispepsia no ul-

${ }^{1}$ Tte. Psicólogo Reservista.

${ }^{2}$ Cte. Psicólogo. Hospital Central de la Defensa Gómez Ulla. Servicio de Psicología Clínica. Madrid. España.

Dirección para correspondencia: José Ignacio Robles Sánchez. Servicio de Psicología Clínica. 5a Planta. Hospital Central de la Defensa "Gómez Ulla". Gta. del Ejército s/n. 28047. Madrid. jrobsan@oc.mde.es

Recibido: 11 de julio de 2013

Aceptado: 21 de octubre de 2013 cerosa, síndrome premenstrual o dolor pélvico crónico, síndrome de hiperventilación, la fatiga crónica, dolor tensional, síndrome de piernas inquietas o sensibilidad química múltiple, entre otros. Los síntomas comunes a estas enfermedades son el dolor, el cansancio, los problemas de sueño y la hiperalgesia generalizada, además de una prevalencia clara en población femenina.

Desde los años 90, se vienen realizando investigaciones acerca de la naturaleza de este grupo de enfermedades. En 1994, Yunus utilizó el concepto inicial de "síndrome disfuncional" para aquellas enfermedades que, como la FM, mostraban los síntomas comunes anteriormente explicados y que carecían de una explicación médica ${ }^{1}$. El habitual solapamiento de los síntomas de unas enfermedades con respecto a otras ha supuesto desde el principio una dificultad a la hora de identificarlas y diagnosticarlas como enfermedades diferenciadas. A día de hoy, pese a que el conocimiento de cada una de ellas se ha especializado, no puede obviarse las similitudes en cuanto a sus manifestaciones $y$, de hecho, los análisis de comorbilidad indican que la relación entre dichas enfermedades es muy estrecha ${ }^{2}$. La actual denominación 


\section{¿Es la Fibromialgia un trastorno depresivo con síntomas somáticos o es un síndrome de...}

de "síndrome de sensibilización central (SSC)", se atribuye a Yunus ${ }^{3}$, quien establece una base etiológica consistente que apunta a que las enfermedades incluidas tienen como origen una hipersensibilidad a los estímulos que recibe el SN. Esta clasificación que incluye expresamente a la FM como SSC ha sido aceptada ampliamente como recogen estudios recientes ${ }^{4-5}$.

Existen diversos estudios que intentan explicar los mecanismos que median en el desarrollo del SSC. Desde un punto de vista fisiopatológico, el dolor crónico puede conducir a cambios neuroplásticos dentro del sistema nervioso central, como consecuencia de la activación persistente de las fibras A delta y C -fibras nerviosas que llevan el impulso nociceptivo a las neuronas del asta dorsal en la médula espinal- estimulando así la liberación de neurotransmisores y neuromoduladores (sustancia P, factor de crecimiento nervioso, neuropéptido relacionado con el gen de la calcitonina, glutamato y aspartato). La influencia de estos neuroquímicos sensibiliza las neuronas que responden a las sensaciones de tacto, presión, temperatura y dolor de tal forma que se convierten en hiperexcitables, respondiendo a niveles más bajos de estímulo nociceptivos (hiperalgesia) así como a estímulos que previamente no eran dolorosos (alodinia) ${ }^{6}$. Además, pueden expandirse las zonas receptivas de los estímulos mediante la activación de neuronas adyacentes, contribuyendo así a la sensación de hiperalgesia. Por otro lado, se ha constatado cómo traumas físicos o las infecciones virales pueden producir la liberación crónica de citoquinas proinflamatorias así como los impulsos dolorosos mantenidos en el tiempo pueden alterar la regulación del SNC tanto en dirección ascendente como descendente?

Otro de los mecanismos que intentan explicar los SSC se asocian al mal funcionamiento del sistema regulador del estrés, el llamado eje hipotálamo-hipofisario-adrenal (HHA). Este mecanismo neuroendocrino es el encargado de transmitir las señales de estrés y relajación desde su recepción a nivel cerebral a través del tejido neuronal hasta sus órganos diana, bien sea órganos inervados o las cápsulas suprarrenales. La alteración de dicho mecanismo está confirmada en diversas enfermedades consideradas SSC. En FM, por ejemplo, las citoquinas proinflamatorias y las IL-6 se ven alteradas en varios estudios y se reconoce que estas pueden alterar el HHA bajo condiciones de estrés produciendo así cambios en el sistema neuroendocrino, como elevación de niveles de serotonina, noradrenalina y dopamina ${ }^{8-10}$. La alteración de estos neurotransmisores pueden dar una explicación para la comprensión tanto de la etiología como de la patogénesis de la FM, si entendemos esta como una enfermedad relacionada con el estrés. La serotonina y noradrenalina median en los procesos de excitación en casos de estrés agudo y son muy importantes para el funcionamiento circadiano del eje HHA ${ }^{11}$, problema tan habitual en personas afectadas de FM y fatiga crónica.

La hormona cortisol también ha sido estudiada por su influencia en los SSC. En el síndrome de intestino irritable (IBS en inglés), adquieren importancia las hormonas corticosteroides en las respuestas de estrés y de ansiedad, que se producen con mayor frecuencia en los afectados por esta enfermedad, siendo responsables, incluso, de una mayor motilidad del colon ${ }^{12-14}$.

En FM, los hallazgos encontrados sobre la hormona cortisol son complejos, existiendo estudios con resultados diferentes, muy probablemente por la metodología empleada. Los primeros estudios encontraban alteraciones del eje HHA con una respuesta anormal de la variación diurna del cortisol plasmático ${ }^{15}$. Estudios más recientes señalan que puede existir una disminución de los niveles basales de cortisol en afectados de $\mathrm{FM}^{16-17}$ aunque existen también estudios que hablan de una normalidad e incluso elevación de los niveles de cortisol ${ }^{18-19}$.

Por último, en el síndrome de fatiga crónica se encuentran igualmente referencias a alteraciones de cortisol en los que los niveles de esta hormona en pacientes se mantienen por debajo de los niveles de población sana ${ }^{20,21}$.

Otras hormonas relacionadas con la alteración del eje HHA en FM son la prolactina y la hormona del crecimiento ${ }^{22,23}$.

En cualquier caso, las alteraciones hormonales debidas al mal funcionamiento del eje HHA en los SSC han sido repetidamente justificadas y los estudios actuales siguen confirmando nuevas relaciones.

El estrés psicológico es uno de los factores intrínsecamente relacionados con el eje HHA y, por tanto, con la patología típica de los SSC. El estrés, la atención intensa o los niveles de vigilancia pueden ser moduladores de las rutas descendentes del SN de la misma forma que las situaciones estresantes presentes o pasadas pueden exacerbar los síntomas de la FM y de los SSC, incluyendo el dolor ${ }^{7,24}$. Este estrés psicológico se puede observar principalmente a través de reacciones de ansiedad y depresión. Si bien no afectan de igual forma a todos los pacientes, ni existe un único perfil psicológico de reacción a los factores psicológicos/cognitivos, dichas reacciones en mayor o menor intensidad son comunes en una proporción importante de personas afectadas por los $\mathrm{SSC}^{25,26}$.

Los rasgos de conducta en estos afectados vienen determinados por ánimo bajo, sentimiento de soledad o tristeza, falta de interés, insomnio y fatiga, entre otros. Además, se da una hipervigilancia ante las señales de dolor y un catastrofismo caracterizado por las expectativas negativas ante el dolor y la enfermedad $^{27}$. Si bien, como hemos visto, los casos de ánimo bajo y niveles relativamente altos de depresión en los pacientes son habituales, varios estudios apuntan a que los estilos cognitivos de los pacientes de FM no son comparables a los afectados por Depresión Mayor, y por tanto, no deberían confundirse los SSC con simples problemas de depresión ${ }^{28}$. Más allá de la simple comorbilidad, algunos estudios apuntan a que el número de síntomas somáticos está relacionado con medidas de estrés emocional ${ }^{29}$.

En cuanto a las relaciones causa-efecto, son abundantes los estudios que demuestran que la sensibilización central puede causar los síntomas, aunque, por otro lado, también existe constancia de que la cronicidad de los síntomas como la ansiedad, el estrés, la depresión y el dolor puede conducir a una sensibilización central?

Por último, la somatización, otro de los rasgos característicos de los $\mathrm{SSC}^{7,30}$, está definida como la expresión física de un problema psicológico ${ }^{31}$. En muchos casos, el problema psicológico podría ser considerado el estrés, la ansiedad y la depresión comentadas anteriormente, demostrando la unión de los mecanismos psicológicos y físicos. Sin embargo, a día de hoy, este término es inadecuado puesto que puede confundirse con el trastorno de somatización incluido dentro de los trastornos somatoformes indicados por el DSM IV-TR. Este es un desorden psiquiátrico relacionado con el síndrome de histeria o de Briquet que nada tiene que ver y que puede dar la impresión de que el problema está en la cabeza del paciente. Esto crearía, y de hecho, crea, además de un error de diagnóstico, una sensación de culpabilidad 
en los pacientes y una hostilidad frente a sus médicos. Por ello, varios informes de expertos aconsejan no incluir ninguna condición de los SSC bajo la clasificación de trastornos somatoformes ni en base al actual DSM IV-TR ni tampoco en base a futuras ediciones del mismo ${ }^{7,32}$. Más bien, este tipo de enfermedades deberían ser consideradas como una condición médica propia, al igual que ocurre con otras enfermedades.

El objetivo de este estudio es poner a prueba un modelo en que se hipotetiza que el impacto de la FM viene dado por la influencia de unas determinadas características de personalidad y de unos valores hormonales alterados, consiguiendo así, apoyar las teorías existentes que atribuyen la FM y los SSC a problemas relacionados con el estrés crónico y con alteraciones hormonales derivadas de esta condición mantenida en el tiempo.

\section{METODO}

\section{Participantes}

Las participantes en el experimento son 48 personas ( 3 hombres y 45 mujeres). Todos los participantes contaban con diagnóstico médico de FM y pertenecían a dos asociaciones diferentes de pacientes de FM (ASAFIMA y FRIDA). Los criterios de exclusión fueron los siguientes: vigilia en las 72 horas previas a las tomas de muestras por causas ajenas a la enfermedad; situación de embarazo; iniciar, modificar o interrumpir durante las se- manas que dura el experimento terapias de cualquier índole que pudieran influir en los resultados experimentales y no asistir a un mínimo de 8 sesiones individuales de la terapia experimental.

El conjunto experimental se dividió en tres grupos (GE1, GE2 y GE3) de forma aleatoria. Mientras que el GE1 se toma como grupo control, los otros dos, GE2 y GE3 se trató de medir los efectos de una terapia con dos tratamientos diferentes. La evaluación de estas formas de terapia no es parte del objetivo del trabajo presente por lo que no nos extenderemos más sobre las mismas.

\section{Materiales}

El CIF (FIQ en inglés) ${ }^{33}$ es un cuestionario de salud específico para los afectados por FM. Actualmente es uno de los instrumentos más utilizados a la hora de medir la salud de afectados por FM. La versión que se utilizó fue la de Esteve-Vives et $\mathrm{al}^{34}$.

El Inventario de Evaluación de la Personalidad (PAI), es un cuestionario de probada validez que mide las variables de personalidad en el campo de la psicología clínica y forense. Su autor es L. Morey.

Fue publicado originalmente en 1991 y revisado recientemente en 2007. Utilizamos la versión larga de este test (344 ítems) por ser la más completa de las dos existentes.

Para la cuantificación de los niveles de cortisol se utilizaron recipientes Salivette ${ }^{\circledR}$ de Sarstedt para la extracción de saliva y

Tabla 1. Informe descriptivo escalas clínicas del PAI muestra 1.

\begin{tabular}{|c|c|c|c|c|c|c|c|c|}
\hline GRUPO & & SOM_1 & ANS_1 & TRA_1 & DEP_1 & MAN_1 & PAR_1 & ESQ_1 \\
\hline \multirow[t]{3}{*}{1} & Media & 83,21 & 70,29 & 68,64 & 77,14 & 53,29 & 59,79 & 59,93 \\
\hline & $\mathrm{N}$ & 14 & 14 & 14 & 14 & 14 & 14 & 14 \\
\hline & Desv. típ. & 7,234 & 9,343 & 9,966 & 13,727 & 9,957 & 11,820 & 23,542 \\
\hline \multirow[t]{3}{*}{2} & Media & 81,31 & 73,06 & 66,19 & 77,81 & 54,94 & 57,69 & 60,38 \\
\hline & $\mathrm{N}$ & 16 & 16 & 16 & 16 & 16 & 16 & 16 \\
\hline & Desv. típ. & 9,857 & 9,574 & 14,171 & 10,784 & 9,277 & 12,965 & 11,224 \\
\hline \multirow[t]{3}{*}{3} & Media & 80,67 & 73,28 & 69,72 & 76,33 & 56,50 & 55,61 & 64,72 \\
\hline & $\mathrm{N}$ & 18 & 18 & 18 & 18 & 18 & 18 & 18 \\
\hline & Desv. típ. & 12,617 & 11,901 & 15,514 & 14,677 & 9,376 & 16,296 & 21,532 \\
\hline \multirow[t]{3}{*}{ Total } & Media & 81,63 & 72,33 & 68,23 & 77,06 & 55,04 & 57,52 & 61,88 \\
\hline & $\mathrm{N}$ & 48 & 48 & 48 & 48 & 48 & 48 & 48 \\
\hline & Desv. típ. & 10,208 & 10,315 & 13,452 & 12,944 & 9,403 & 13,830 & 19,136 \\
\hline GRUPO & & LIM_1 & ANT_1 & AGR_1 & ICN_1 & INF_1 & IMN_1 & IMP_1 \\
\hline \multirow[t]{3}{*}{1} & Media & 63,64 & 51,79 & 42,21 & 62,93 & 55,00 & 63,43 & 46,07 \\
\hline & $\mathrm{N}$ & 14 & 14 & 14 & 14 & 14 & 14 & 14 \\
\hline & Desv. típ. & 10,616 & 8,763 & 6,447 & 8,695 & 10,183 & 10,704 & 11,486 \\
\hline \multirow[t]{3}{*}{2} & Media & 66,31 & 51,75 & 43,50 & 58,94 & 53,44 & 66,00 & 44,06 \\
\hline & $\mathrm{N}$ & 16 & 16 & 16 & 16 & 16 & 16 & 16 \\
\hline & Desv. típ. & 8,023 & 5,814 & 5,727 & 11,210 & 9,077 & 9,973 & 9,876 \\
\hline \multirow[t]{3}{*}{3} & Media & 66,22 & 55,56 & 44,61 & 58,61 & 53,56 & 66,44 & 43,22 \\
\hline & $\mathrm{N}$ & 18 & 18 & 18 & 18 & 18 & 18 & 18 \\
\hline & Desv. típ. & 11,690 & 5,742 & 7,269 & 7,445 & 10,629 & 13,921 & 12,374 \\
\hline \multirow[t]{3}{*}{ Total } & Media & 65,50 & 53,19 & 43,54 & 59,98 & 53,94 & 65,42 & 44,33 \\
\hline & $\mathrm{N}$ & 48 & 48 & 48 & 48 & 48 & 48 & 48 \\
\hline & Desv. típ. & 10,129 & 6,884 & 6,484 & 9,206 & 9,814 & 11,629 & 11,153 \\
\hline
\end{tabular}


¿Es la Fibromialgia un trastorno depresivo con síntomas somáticos o es un síndrome de...

Tabla 2. Informe descriptivo subescalas clínicas del PAI muestra 1.

\begin{tabular}{|c|c|c|c|c|c|c|c|c|c|c|c|}
\hline $\mathbf{G r}$ & & SOMC & SOMS & SOMH & ANSC & ANSE & ANSF & TRAE & DEPC & DEPE & DEPF \\
\hline \multirow[t]{3}{*}{1} & Media & 85,29 & 76,29 & 76,64 & 68,79 & 67,64 & 69,21 & 68,50 & 69,71 & 77,43 & 70,50 \\
\hline & $\mathrm{N}$ & 14 & 14 & 14 & 14 & 14 & 14 & 14 & 14 & 14 & 14 \\
\hline & Desv. típ. & 13,505 & 5,967 & 8,445 & 7,084 & 10,775 & 15,463 & 11,979 & 12,048 & 16,887 & 12,107 \\
\hline \multirow[t]{3}{*}{2} & Media & 80,44 & 78,56 & 74,00 & 70,19 & 66,38 & 75,94 & 69,00 & 70,19 & 74,69 & 74,69 \\
\hline & $\mathrm{N}$ & 16 & 16 & 16 & 16 & 16 & 16 & 16 & 16 & 16 & 16 \\
\hline & Desv. típ. & 13,692 & 10,139 & 12,006 & 8,416 & 10,006 & 13,709 & 14,904 & 10,458 & 13,032 & 10,203 \\
\hline \multirow[t]{3}{*}{3} & Media & 81,17 & 76,56 & 73,22 & 69,11 & 70,61 & 72,00 & 64,17 & 68,44 & 74,28 & 71,50 \\
\hline & $\mathrm{N}$ & 18 & 18 & 18 & 18 & 18 & 18 & 18 & 18 & 18 & 18 \\
\hline & Desv. típ. & 14,750 & 11,299 & 13,808 & 12,653 & 13,311 & 12,315 & 14,076 & 13,747 & 15,533 & 12,368 \\
\hline \multirow[t]{3}{*}{ Total } & Media & 82,13 & 77,15 & 74,48 & 69,38 & 68,33 & 72,50 & 67,04 & 69,40 & 75,33 & 72,27 \\
\hline & $\mathrm{N}$ & 48 & 48 & 48 & 48 & 48 & 48 & 48 & 48 & 48 & 48 \\
\hline & Desv. típ. & 13,902 & 9,480 & 11,695 & 9,734 & 11,471 & 13,722 & 13,688 & 12,000 & 14,907 & 11,500 \\
\hline Gr & & ESQP & ESQS & ESQA & LIME & LIMI & LIMP & LIMA & PARH & PARP & PARR \\
\hline \multirow[t]{3}{*}{1} & Media & 59,07 & 54,86 & 71,86 & 64,50 & 66,29 & 59,14 & 51,36 & 56,86 & 55,14 & 62,43 \\
\hline & $\mathrm{N}$ & 14 & 14 & 14 & 14 & 14 & 14 & 14 & 14 & 14 & 14 \\
\hline & Desv. típ. & 15,843 & 11,954 & 13,850 & 11,928 & 11,083 & 8,883 & 10,717 & 9,054 & 11,967 & 12,157 \\
\hline \multirow[t]{3}{*}{2} & Media & 51,94 & 51,19 & 70,69 & 66,25 & 67,06 & 61,94 & 55,25 & 58,44 & 53,56 & 57,38 \\
\hline & $\mathrm{N}$ & 16 & 16 & 16 & 16 & 16 & 16 & 16 & 16 & 16 & 16 \\
\hline & Desv. típ. & 9,241 & 11,065 & 11,948 & 8,473 & 8,948 & 10,440 & 11,829 & 14,014 & 11,466 & 12,569 \\
\hline \multirow[t]{3}{*}{3} & Media & 55,11 & 51,50 & 68,61 & 66,28 & 64,83 & 61,28 & 57,39 & 52,89 & 54,44 & 56,56 \\
\hline & $\mathrm{N}$ & 18 & 18 & 18 & 18 & 18 & 18 & 18 & 18 & 18 & 18 \\
\hline & Desv. típ. & 11,999 & 10,853 & 12,650 & 14,340 & 12,196 & 12,924 & 15,042 & 13,651 & 14,492 & 18,286 \\
\hline \multirow[t]{3}{*}{ Total } & Media & 55,21 & 52,38 & 70,25 & 65,75 & 66,00 & 60,88 & 54,92 & 55,90 & 54,35 & 58,54 \\
\hline & $\mathrm{N}$ & 48 & 48 & 48 & 48 & 48 & 48 & 48 & 48 & 48 & 48 \\
\hline & Desv. típ. & 12,524 & 11,127 & 12,584 & 11,717 & 10,689 & 10,880 & 12,823 & 12,597 & 12,567 & 14,789 \\
\hline
\end{tabular}

para el análisis en laboratorio de estas muestras, se utilizó el kit DEMEDITEC Cortisol ELISA.

Para el análisis y cruce de los datos aportados por los registros psicológicos y hormonales empleamos el programa informático SPSSPC ver. 15 para Windows con licencia de uso para la UCM y programa IBM SPSS ${ }^{\circledR} \operatorname{Amos}^{\mathrm{TM}} 19$.

\section{Procedimiento}

Antes de la toma de la primera muestra, los participantes fueron informados de la naturaleza y condiciones del experimento, remarcando la voluntariedad de la participación así como del compromiso de confidencialidad por parte del investigador. Los sujetos leyeron y firmaron un consentimiento informado acerca de sus datos personales. El estudio respeta la Declaración de Helsinki de la AMM sobre investigaciones médicas en seres humanos y sus posteriores revisiones. Por último, los participantes cumplimentaron un cuestionario sobre aspectos personales que podrían ser utilizados como criterios de exclusión o para aclarar algún aspecto no cubierto por las muestras de cortisol o los tests.

Se tomaron muestras en dos momentos diferentes mediante el cuestionario CIF, el cuestionario PAI y la muestra de saliva. La primera muestra (21-1-2012) tuvo lugar antes del comienzo del período experimental de 10 semanas para medir las condiciones habituales de los sujetos. La segunda muestra (31-3-2012) se realizó al finalizar la fase para comparar a los sujetos con sus medidas previas y medir así la influencia del tratamiento experimental. Los registros se tomaron de forma conjunta en el mismo lugar y a la misma hora en ambas ocasiones para evitar posibles variaciones debidas al ritmo circadiano o a otras variables.

Una vez extraídas las muestras de cortisol, se mantuvieron bajo cadena de frío mediante congelación hasta su posterior análisis en los laboratorios de Psicobiología de la Facultad de Psicología de la Universidad Complutense de Madrid por personal de esa institución.

\section{RESULTADOS}

\section{Resultados PAI Primera Muestra}

A continuación, mostramos los análisis descriptivos obtenidos de la prueba PAI en la primera muestra. En la tabla 1 aparecen todas las escalas clínicas evaluadas por el PAI y en la tabla 2 aparecen las subescalas clínicas del PAI que han expresado resultados significativos.

La escala Somatización (SOM) refleja un grado de preocupación importante por la salud física y un elevado nivel de quejas somáticas. Se observan leves diferencias entre las subescalas (véase Tabla 2).

La escala Ansiedad (ANS) muestra una puntuación significativa (72.33T) señalando un estado de preocupación y/o tensión constantes. Todas la subescalas se mantienen en medidas similares, aunque la ansiedad fisiológica (ANSF) marca de manera más significativa $(72,50 \mathrm{~T})$. 
La escala Depresión (DEP) obtiene puntuaciones significativas. Todas las subescalas se mantienen altas, especialmente la subescala depresión emocional (DEPE).

Trastornos relacionados con la ansiedad (TRA) obtiene niveles elevados. En esta escala se aprecian diferencias por subescalas: los niveles son normales en trastorno obsesivocompulsivo (TRAO) y en fobias (TRAF). En cambio las puntuaciones en la subescala Estrés postraumático (TRAE) son significativamente altas $(67,04 \mathrm{~T})$.

La variable Paranoidismo (PAR) muestra normalidad en cuanto a síntomas de los trastornos paranoide. Únicamente podemos encontrar una ligera tendencia hacia el Resentimiento y la desconfianza (PARR) en uno de los grupos experimentales.

La variable Esquizofrenia (ESQ) obtiene en conjunto una puntuación no significativa $(61,88 \mathrm{~T})$. Sin embargo existen grandes diferencias entre las subescalas. La subescala Alteración del pensamiento (ESQA) muestra valores significativos. En cambio, Experiencias psicóticas (ESQP) e Indiferencia social (ESQS) obtienen puntuaciones bajas y dentro de la normalidad.

La escala Rasgos límites (LIM) puntúa 65,50T indicando niveles significativos. Encontramos diferencias entre las subescalas: LIME y LIMI obtienen puntuaciones de 65,75 T y $66 \mathrm{~T}$ expresando cierta tendencia a la inestabilidad emocional y a un grado elevado de sensibilidad emocional, por un lado, y a las dudas por aspectos vitales y sensación de vacío, por otro. En cambio las subescalas de relaciones interpersonales problemáticas (LIMP) y autolesiones (LIMA) puntúan bajas y dentro de la normalidad.

La variable Rasgos antisociales (ANT) indica niveles normales de empatía y socialización. Igualmente la escala Agresividad (AGR) expresa un control razonable sobre la expresión de la ira y hostilidad. En cuanto a la variable Manía (MAN), las puntuaciones son normales (55T), propias de personas alejadas de manía o hipomanía.

Los marcadores de fiabilidad indican normalidad en todas sus variables: Inconsistencia (ICN), Infrecuencia (INF), Impresión negativa (IMN) e Impresión positiva (IMP), eliminando así cualquier duda especialmente sobre la simulación o exageración de síntomas por parte de los pacientes o la no comprensión de los ítems del test. Pese a que la puntuación en Impresión negativa (IMN) aparece ligeramente elevada $(65,42 \mathrm{~T})$, los valores indican que no existe un impacto apreciable (menor a $72 \mathrm{~T}$ ). Quizá estos valores se deban a la posible correlación con las escalas clínicas.

Tabla 3. Informe descriptivo resultados del CIF y cortisol ( $\mathrm{ng} / \mathrm{ml}$ ) en la muestra 1.

\begin{tabular}{|cccc|}
\hline GRUPO & & CIF_1 & CORT_1 \\
\hline 1 & Media & 69,1214 & 6,9209 \\
& $\mathrm{~N}$ & 14 & 14 \\
& Desv. típ. & 16,47398 & 2,81722 \\
2 & Media & 65,3431 & 8,1947 \\
& $\mathrm{~N}$ & 16 & 16 \\
& Desv. típ. & 17,39746 & 5,65801 \\
& Media & 62,9861 & 7,0267 \\
& $\mathrm{~N}$ & 18 & 18 \\
& Desv. típ. & 20,47889 & 2,12212 \\
& Media & 65,5613 & 7,3851 \\
& N & 48 & 48 \\
& Desv. típ. & 18,15729 & 3,79178 \\
\hline
\end{tabular}

\section{Resultados CIF y cortisol Primera Muestra}

El estudio realizado por Esteve-Vives et $\mathrm{al}^{34}$ sobre el que se basa este Cuestionario de Impacto de la Fibromialgia encuentra una media de 70,5 puntos, lo cual indica una media alta de impacto de FM en los sujetos de su estudio. Nuestro estudio, según muestra la tabla 3, obtiene unos resultados del CIF similares con una puntuación media de los tres grupos de 65,56 en el conjunto de sujetos, advirtiendo de la relevancia de la enfermedad en la calidad de vida habitual de los afectados. Los resultados de las muestras son similares entre los grupos 1,2 y 3 .

Los niveles de cortisol encontrados en el conjunto de sujetos en la primera muestra es de $7,381 \mathrm{nmol} / 1$, tal y como muestra la tabla 3, con ligeras diferencias entre los grupos, siendo el grupo 2 el que muestra los niveles más altos, sin significación estadística.

\section{Resultados PAI segunda muestra}

Aunque los datos expresados en la segunda muestra están alterados por la influencia de un tratamiento experimental en los grupos 2 y 3, podemos observar (tabla 4) que las puntuaciones siguen manteniéndose altas en las escalas Somatización $(77,10 \mathrm{~T})$, Ansiedad $(65,60 \mathrm{~T})$ y Depresión $(69,17 \mathrm{~T})$, por lo que someteremos estas escalas a un análisis factorial posterior.

\section{Resultados CIF y cortisol segunda muestra}

Según muestra la tabla 5, la media del CIF en el conjunto de los individuos no es muy alta, tan solo 43,80 puntos. Pero si observamos por grupos las medias, encontramos que el grupo 1 (control) obtiene una puntuación muy alta en dicho test $(68,18)$ similar a la primera muestra, mientras que en los grupos 2 y 3 las puntuaciones disminuyen hasta los 39,24 y 28,89 respectivamente, indicando un bajo impacto de la FM en la calidad de vida de los sujetos y una disminución de puntuaciones frente a la primera muestra.

Los resultados de cortisol obtenidos en la segunda toma muestran diferencias entre los grupos siendo el grupo 1 el que mayor tasa de cortisol obtiene $(6,8521 \mathrm{ng} / \mathrm{ml})$. El grupo 2 obtiene la tasa más baja de los tres, $5,9208 \mathrm{ng} / \mathrm{ml}$ y el grupo 3 obtiene unos resultados intermedios de $6,0007 \mathrm{ng} / \mathrm{ml}$.

\section{Comparación de los grupos}

Realizado el ANOVA de un factor para detectar diferencias entre los resultados de la primera muestra de los grupos encontramos que las medias de los tres grupos son similares. Sin embargo, el estadístico de Levene detectó falta de homogeneidad en la variable procedencia, sexo y cortisol, por lo que utilizamos la prueba de Kruskal-Wallis como medida no paramétrica encontrando una igualdad entre los resultados salvo la procedencia que, por razones prácticas no se pudo distribuir aleatoriamente.

En el ANOVA de la segunda muestra, los resultados fueron similares, debiendo utilizar, de nuevo la prueba de Kruskal-Wallis y encontrando diferencias significativas entre los grupos en la variable procedencia y en el CIF en la muestra 2. 
¿Es la Fibromialgia un trastorno depresivo con síntomas somáticos o es un síndrome de...

Tabla 4. Informe descriptivo escalas clínicas del PAI muestra 2.

\begin{tabular}{|c|c|c|c|c|c|c|c|c|}
\hline GRUPO & & SOM_2 & ANS_2 & TRA_2 & DEP_2 & MAN_2 & PAR_2 & ESQ_2 \\
\hline \multirow[t]{3}{*}{1} & Media & 81,57 & 67,93 & 64,07 & 74,57 & 49,29 & 58,86 & 67,00 \\
\hline & $\mathrm{N}$ & 14 & 14 & 14 & 14 & 14 & 14 & 14 \\
\hline & Desv. típ. & 11,843 & 11,906 & 13,635 & 16,266 & 9,972 & 11,044 & 23,642 \\
\hline \multirow[t]{3}{*}{2} & Media & 76,69 & 65,94 & 61,13 & 71,06 & 50,13 & 55,25 & 60,25 \\
\hline & $\mathrm{N}$ & 16 & 16 & 16 & 16 & 16 & 16 & 16 \\
\hline & Desv. típ. & 10,117 & 9,637 & 11,117 & 10,453 & 10,500 & 11,042 & 24,447 \\
\hline \multirow[t]{3}{*}{3} & Media & 74,00 & 63,50 & 57,72 & 63,28 & 51,61 & 51,56 & 54,00 \\
\hline & $\mathrm{N}$ & 18 & 18 & 18 & 18 & 18 & 18 & 18 \\
\hline & Desv. típ. & 12,834 & 10,864 & 10,632 & 14,029 & 6,617 & 12,940 & 10,307 \\
\hline \multirow[t]{3}{*}{ Total } & Media & 77,10 & 65,60 & 60,71 & 69,17 & 50,44 & 54,92 & 59,88 \\
\hline & $\mathrm{N}$ & 48 & 48 & 48 & 48 & 48 & 48 & 48 \\
\hline & Desv. típ. & 11,863 & 10,718 & 11,773 & 14,228 & 8,915 & 11,925 & 20,302 \\
\hline GRUPO & & LIM_2 & ANT_2 & AGR_2 & ICN_2 & INF_2 & IMN_2 & IMP_2 \\
\hline \multirow[t]{3}{*}{1} & Media & 59,86 & 48,71 & 39,21 & 57,57 & 53,21 & 63,57 & 48,36 \\
\hline & $\mathrm{N}$ & 14 & 14 & 14 & 14 & 14 & 14 & 14 \\
\hline & Desv. típ. & 12,811 & 7,477 & 5,041 & 6,098 & 7,678 & 13,369 & 12,326 \\
\hline \multirow[t]{3}{*}{2} & Media & 61,94 & 49,25 & 41,44 & 57,63 & 53,19 & 59,00 & 45,94 \\
\hline & $\mathrm{N}$ & 16 & 16 & 16 & 16 & 16 & 16 & 16 \\
\hline & Desv. típ. & 8,737 & 4,726 & 5,341 & 10,032 & 6,997 & 11,866 & 7,945 \\
\hline \multirow[t]{3}{*}{3} & Media & 57,72 & 52,50 & 42,61 & 55,67 & 56,11 & 60,11 & 49,33 \\
\hline & $\mathrm{N}$ & 18 & 18 & 18 & 18 & 18 & 18 & 18 \\
\hline & Desv. típ. & 10,465 & 7,556 & 4,984 & 8,268 & 8,547 & 10,363 & 9,305 \\
\hline \multirow[t]{3}{*}{ Total } & Media & 59,75 & 50,31 & 41,23 & 56,88 & 54,29 & 60,75 & 47,92 \\
\hline & $\mathrm{N}$ & 48 & 48 & 48 & 48 & 48 & 48 & 48 \\
\hline & Desv. típ. & 10,610 & 6,798 & 5,203 & 8,248 & 7,771 & 11,696 & 9,780 \\
\hline
\end{tabular}

En cualquier caso se puede decir que la comparación entre los grupos en ambas muestras indica que no hay diferencias importantes.

\section{Análisis de la primera muestra}

\section{Análisis factorial exploratorio}

Mediante este análisis se intenta comprobar si existe o no agrupación de variables y de qué modo se agrupan para explicar la varianza común.

Tabla 5. Informe descriptivo CIF y cortisol ( $\mathrm{ng} / \mathrm{ml})$ muestra 2.

\begin{tabular}{|cccc|}
\hline GRUPO & & CIF_2 & CORT_2 \\
\hline 1 & Media & 68,1800 & 6,8521 \\
& $\mathrm{~N}$ & 14 & 14 \\
& Desv. típ. & 15,36748 & 2,81551 \\
& Media & 39,2400 & 5,9208 \\
& $\mathrm{~N}$ & 16 & 16 \\
& Desv. típ. & 15,50273 & 3,01748 \\
& Media & 28,8978 & 6,0007 \\
& $\mathrm{~N}$ & 18 & 18 \\
& Desv. típ. & 16,58123 & 3,53015 \\
& Media & 43,8025 & 6,2224 \\
& N & 48 & 48 \\
& Desv. típ. & 22,59753 & 3,12633 \\
\hline
\end{tabular}

En el Análisis Factorial de la primera muestra, la medida de Kaiser-Meyer-Olkin (KMO) nos muestra una adecuación aceptable $(\mathrm{KMO}=0,691)$, mientras que el análisis de esfericidad de Bartlett indica un excelente nivel de adecuación (sig $=0,000)$.

Encontramos que de los cuatro factores que explican el $73 \%$ de la varianza común, el primero explica el $43 \%$ de la misma y está conformado por las siguientes variables: escalas del PAI (Somatización, Ansiedad, Depresión, Trastornos Relacionados con la Ansiedad, Paranoidismo, Esquizofrenia, Rasgos Límites e Impresión Negativa), Cuestionario de Impacto de la Fibromialgia y Cortisol.

\section{Análisis factorial confirmatorio}

Intentamos encontrar un modelo que explique la fibromialgia en función de los resultados obtenidos en el análisis factorial exploratorio de la primera muestra. Para ello, ponemos a prueba un modelo que relaciona los factores de personalidad medidos por el PAI, los resultados de cortisol recogidos y la influencia de estos dos factores en la calidad de vida, medida esta por los resultados obtenidos en el CIF.

Según se indica en el "Path diagram" de la Fig.1, lo que explica la FM en la primera muestra (entendida la FM por el índice de impacto de la enfermedad en la calidad de vida, medida por el cuestionario CIF) es la personalidad medida por el PAI, con Somatización, Ansiedad, Depresión, Trastornos Relacionados con la Ansiedad, Paranoia, Esquizofrenia, Rasgos Límites y escala de Impresión negativa. El cortisol no tiene, apenas, influencia. 


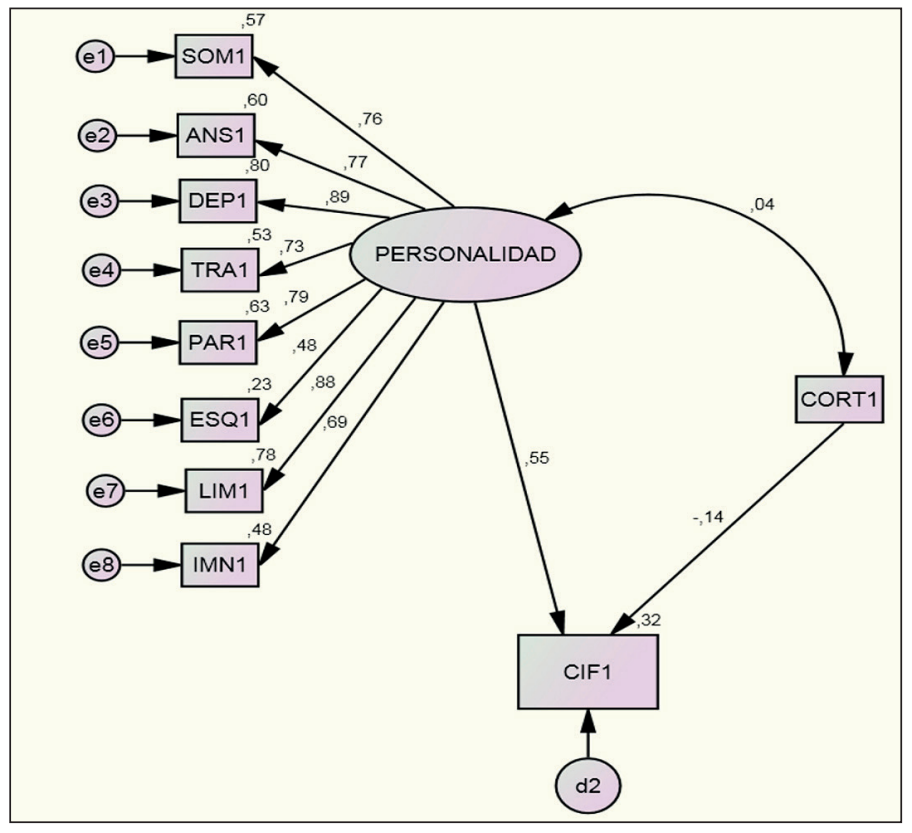

Figura 1. Path diagram primera muestra.

En cuanto al análisis de nuestro modelo de la muestra 1, podemos decir que el índice de mínima discrepancia (CMIN) se mantiene dentro de unos parámetros de ajuste, al ser $\mathrm{P}(0,005)$ superior a 0 . Según refleja la tabla 6 , los valores de GFI y AGFI se encuentran próximos a $1 \mathrm{y}$, por tanto, indican un buen ajuste del modelo. El RMSEA marca un valor superior a 0,05. Según este indicador, los datos no se ajustarían al modelo. Puede que este dato se deba a la complejidad del modelo o al tamaño de la muestra. El AIC, el BCC, el BIC y el CAIC muestran valores menores de nuestro modelo con respecto a los otros, lo cual es señal de mejor ajuste. Lo mismo sucede con ECVI. La adecuación del tamaño de la muestra queda en evidencia por el parámetro Hoelter. Para que la muestra fuera adecuada en tamaño, el valor debería ascender a 200, mientras que apenas llega a 39 y 45. La falta de adecuación en este sentido, pueden haber forzado los valores del RMSEA.

Podemos decir, por tanto, que los datos de esta primera muestra confirman el modelo propuesto. Esto es, que la FM viene condicionada por las variables de personalidad del PAI (Somatización, Ansiedad, Depresión, Trastornos Relacionados con la Ansiedad, Paranoia, Esquizofrenia e Impresión negativa), pero no se demuestra una relación con el cortisol.

\section{Análisis de la segunda muestra}

\section{Análisis factorial exploratorio}

En el segundo análisis factorial exploratorio encontramos también una buena adecuación de la medida $\operatorname{KMO}(0,691)$ y un excelente resultado en la prueba de Bartlett $(\mathrm{sig}=0,000)$.

En este caso, son cinco factores los que explican el $76 \%$ de la varianza común. El primero explica el 37,24\%, y está conformado por Somatización, Ansiedad, Depresión, Trastornos Relacionados con la Ansiedad, Paranoia, Esquizofrenia, Rasgos Límites.

Tabla 6. Análisis confirmatorio muestra 1.

\begin{tabular}{|l|l|l|l|l|l|}
\hline CMIN & NPAR & CMIN & DF & $P$ & $\begin{array}{l}\text { CMIN/ } \\
\text { DF }\end{array}$ \\
\hline Model & 21 & 58,651 & 34 & 0,05 & 1,725 \\
\hline Default Model & 25 &, 000 & 0 & & \\
\hline Saturated Model & 55 & & & \\
\hline Independence Model & 10 & 300,323 & 45 &, 000 & 6,674 \\
\hline
\end{tabular}

\begin{tabular}{|l|l|l|l|l|}
\hline AIC & AIC & BCC & BIC & CAIC \\
\hline Model & 100,651 & 113,484 & 139,946 & 160,946 \\
\hline Default Model & 110,000 & 143,611 & 212,916 & 267,916 \\
\hline Saturated Model & 320,313 & 326,434 & 339,035 & 349,035 \\
\hline Independence Model & 32, & &
\end{tabular}

\begin{tabular}{|l|l|l|l|l|}
\hline RMR, GFI & RMR & GFI & AGFI & PGFI \\
\hline Model & $\mathbf{9 , 6 1 9}$ &, 826 &, 719 &, 511 \\
\hline Default Model &, 000 & 1,000 & & \\
\hline Saturated Model & 70,260 &, 317 &, 165 &, 259 \\
\hline Independence Model & 765 \\
\hline
\end{tabular}

\begin{tabular}{|l|l|l|l|l|}
\hline ECVI & ECVI & LO90 & HI90 & MECVI \\
\hline Model & 2,142 & 1,771 & 2,679 & 2,415 \\
\hline Default Model & 2,340 & 2,340 & 2,340 & 3,056 \\
\hline Saturated Model & 6,815 & 5,727 & 8,063 & 6,945 \\
\hline Independence Model &
\end{tabular}

\begin{tabular}{|l|l|l|l|l|}
\hline RMSEA \\
\hline Model & RMSEA & LO90 & HI90 & PCLOSE \\
\hline Default Model &, 124 &, 067 &, 177 &, 022 \\
\hline Independence Model &, 347 &, 311 &, 385 &, 000 \\
\hline
\end{tabular}

\begin{tabular}{|c|c|c|}
\hline \multicolumn{3}{|l|}{ HOELTER } \\
\hline Model & HOELTER.0S & HOELTER.01 \\
\hline Default Model & 39 & 45 \\
\hline Independence Model & 10 & 11 \\
\hline Minimization: & .016 & \\
\hline Miscellaneous: & 297 & \\
\hline Bootstrap: & .000 & \\
\hline Total: &, 313 & \\
\hline
\end{tabular}

AGFI: Adjusted Goodness of Fit Index; AIC: Akaike Information Criterion; BCC: Browne-Cudeck Criterion, BIC: Bayes Information Criterion; CAIC: Consistent Akaike Information Criterion; ECVI: Expected cross-validation Index; GFI: Goodness of Fit Index; RMSEA: Root Mean Square Error of Approximation. 
¿Es la Fibromialgia un trastorno depresivo con síntomas somáticos o es un síndrome de...

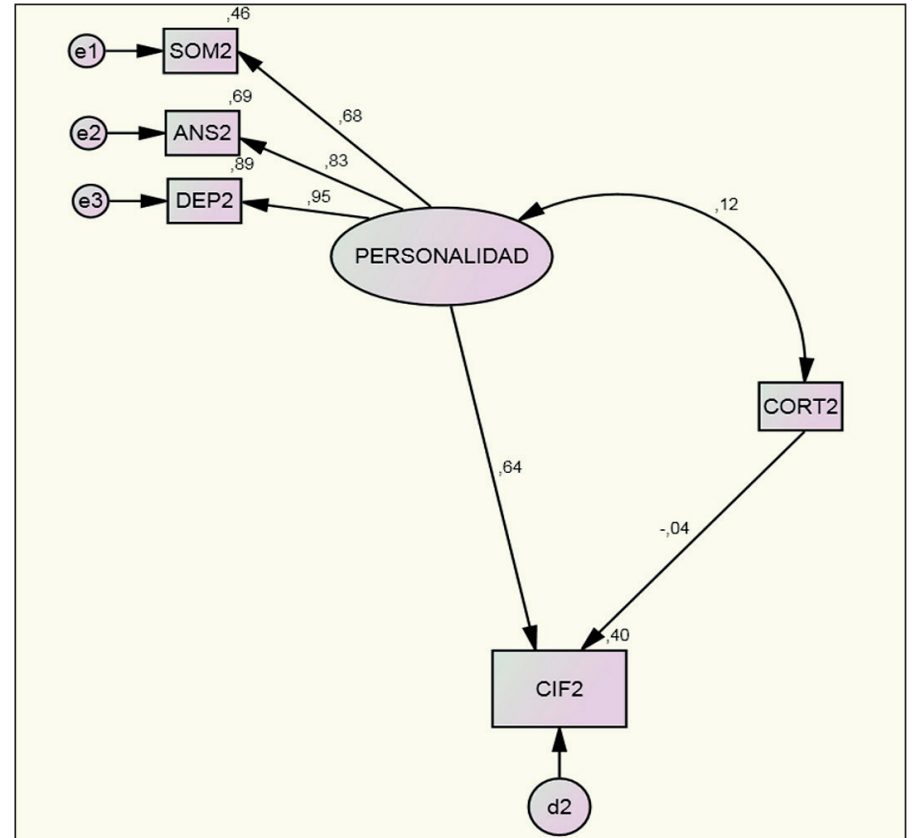

Figura 2. Path diagram segunda muestra, primer modelo.

\section{Análisis factorial confirmatorio}

En el análisis confirmatorio de este factor hemos considerado dos modelos diferentes. En un primer modelo proponemos que la FM, medida por su impacto en la calidad de vida de los pacientes mediante el CIF tiene que ver únicamente con tres factores de personalidad medidos por el PAI (Somatización, Ansie-

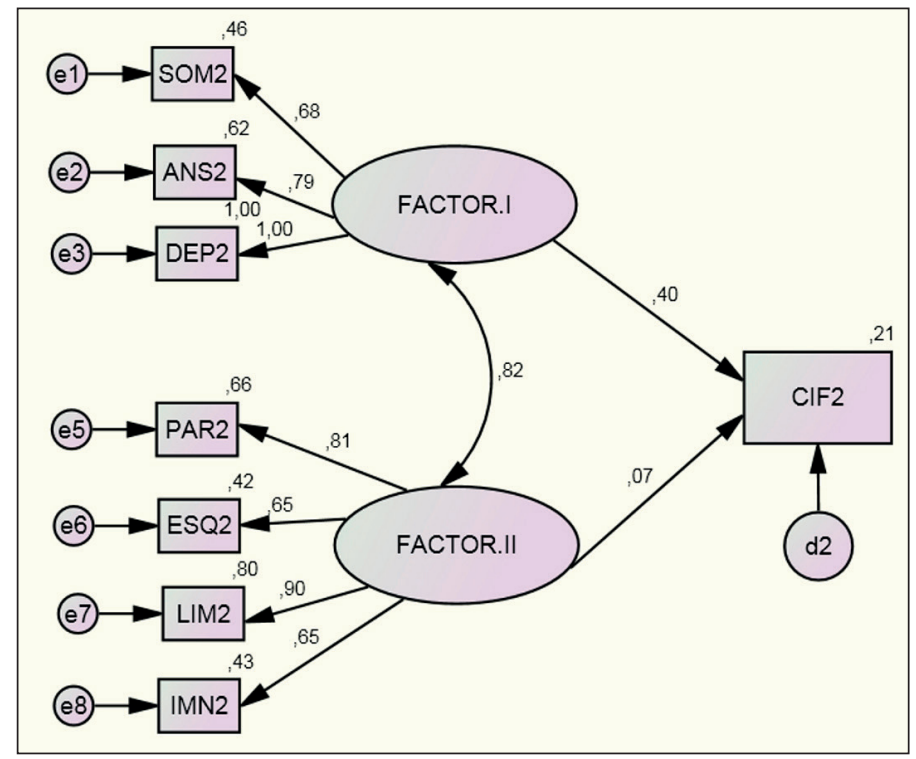

Figura 3. Path diagram segunda muestra segundo modelo.

dad y Depresión) y con el cortisol. De nuevo, encontramos que estos tres factores -y en especial el de la depresión, sí explican la personalidad como causalidad en el CIF, mientras que el cortisol (.04) no influye apenas.

En este caso (tabla 7) el CMIN muestra un ajuste casi perfecto, con una P de 0,952. Los marcadores GFI y AGFI también muestran un ajuste óptimo con valores muy cercanos al 1. El RMSEA es de 0,00 con lo cual también nos habla de la excelente bondad de ajuste del modelo. El AIC, el BCC, BIC y CAIC

Tabla 7. Análisis confirmatorio muestra 2, primer modelo

\begin{tabular}{|c|c|c|c|c|c|}
\hline \multicolumn{6}{|l|}{ CMIN } \\
\hline Model & NPAR & CMIN & DF & $P$ & $\begin{array}{l}\text { CMIN } \\
\text { DF }\end{array}$ \\
\hline Default Model & 11 & .691 & 4 & .952 & 173 \\
\hline Saturated Model & 15 &, 000 & 0 & & \\
\hline Independence Model & 5 & 93,619 & 10 & .000 & 9,362 \\
\hline
\end{tabular}

\begin{tabular}{|l|l|l|l|l|}
\hline AIC & AIC & BCC & BIC & CAIC \\
\hline Model & 22,691 & 25,910 & 43,274 & 54,274 \\
\hline Default Model & 30,000 & 34,390 & 58,068 & 73,068 \\
\hline Saturated Model & 103,619 & 105,082 & 112,975 & 117,975 \\
\hline Independence Model & & & &
\end{tabular}

\begin{tabular}{|l|l|l|l|l|}
\hline RMR, GFI & RMR & GFI & AGFI & PGFI \\
\hline Model & 3,517 &, 994 &, 978 &, 265 \\
\hline Default Model &, 000 & 1,000 & & \\
\hline Saturated Model & 79,841 &, 531 &, 296 &, 354 \\
\hline Independence Model & 79 \\
\hline
\end{tabular}

\begin{tabular}{|l|l|l|l|l|}
\hline ECVI & ECVI & LO90 & HI90 & MECVI \\
\hline Model &, 483 & .553 &, 553 &, 551 \\
\hline Default Model & .638 & .638 &, 638 &, 732 \\
\hline Saturated Model & 2,205 & 1,623 & 2,945 & 2,236 \\
\hline Independence Model & & &
\end{tabular}

\begin{tabular}{|l|l|l|l|l|}
\hline \multicolumn{5}{|l|}{ RMSEA } \\
\hline Model & RMSEA & 1090 & HI90 & PCLSE \\
\hline Default Model &, 000 &, 000 &, 000 &, 961 \\
\hline Independence Model &, 422 &, 346 &, 502 &, 000 \\
\hline
\end{tabular}

\begin{tabular}{|l|c|c|}
\hline \multicolumn{3}{|l|}{ HOELTER } \\
\hline Model & HOELTER.05 & HOELTER.01 \\
\hline Default Model & \multicolumn{1}{|c|}{646} & 904 \\
\hline Independence Model & 10 & 12 \\
\hline Minimization: &, 016 & \\
Miscellaneous: & .187 & \\
Bootstrap: &, 000 & \\
Total: &, 203 & \\
\cline { 1 - 2 } & &
\end{tabular}


muestran los valores más bajos respecto al modelo saturado y al modelo independiente, mostrando, de nuevo, un buen ajuste. Lo mismo ocurre con el ECVI.

Por último, probamos la validez de un modelo compuesto esta vez por dos factores. Este tercer modelo descompone las variables de personalidad en dos factores, uno formado por Somatización, Ansiedad y Depresión y otro definido por Paranoidismo, Esquizofrenia, Rasgos Límites e Impresión Negativa. El factor I es el que más peso tiene sobre la FM, (.40) siendo la Depresión la variable más importante, mientras que los componentes del factor II (PAR, ESQ, LIM e IMN) tienen una correlación escasa (.07), sobre la FM medida por el impacto que causa.

El indicador CMIN (tabla 8) muestra cierto ajuste del modelo con una P de 0,039. Los valores de FFI y AGFI se acercan a 1, lo cual es señal de un buen ajuste. El RMSEA es de 0,116 equivalente a un mal ajuste. Sin embargo, las causas pueden deberse de nuevo al tamaño de la muestra, como indica el parámetro Hoelter, que debiera llegar a 200 y en cambio se mantiene lejano de dicha puntuación. AIC, BCC, BIC y CAIC marcan valores menores a los modelos saturado e independencia, expresando, por tanto, un buen ajuste.

Por ello, podemos decir que este tercer modelo confirma de nuevo la importancia de los factores de personalidad sobre la FM expresada mediante el CIF, aunque no de una forma tan notoria como el segundo modelo. El modelo 2 es el que mejor explica el impacto que causa la enfermedad en la vida de los pacientes, medido por el CIF, en función de tres variables: Somatización, Ansiedad y Depresión.

\section{DISCUSIÓN}

Las medidas de personalidad recogidas mediante el PAI en condiciones normales de los sujetos experimentales afectados de FM muestran unas características psicológicas muy determinadas.

Los valores de estos sujetos en la escala Somatización (SOM) son muy superiores a los considerados normales, que en el caso de ancianos y pacientes enfermos se encuentran entre 60 y 69T, y en el caso de personas con pocas quejas o sanas no supera los 59T. Los resultados obtenidos describen a estos sujetos afectados por FM como muy preocupados por la salud física y con abundantes quejas corporales. Cobran especial relevancia las quejas acerca de alteraciones motoras y de percepciones sensoriales (SOMC) experimentadas por los afectados, especialmente en cuanto a limitación de capacidad de movimientos y las sensaciones dolorosas. También se encuentran notables quejas de cansancio o de apreciación de mala salud muy habituales (SOMS). Estos datos encajan con los resultados obtenidos por el CIF que miden precisamente el grado de incapacidad física y las limitaciones en las actividades diarias como caminar, limpiar, etc.

Por tanto, podemos decir que el test recoge la habitual percepción tanto de los propios pacientes como de los especialistas y personas cercanas, de que estas personas expresan quejas físicas de forma habitual y que estas conllevan una reducción muy importante de la calidad de vida.

Sin embargo, tal y como proponen varios autores ${ }^{7,31}$, esta definición de Somatización puede llevar a confusión, dando la impresión de que las quejas son expresión de una alteración psiquiátrica, cuando, en muchos casos, nada tiene que ver. Por ello, queremos redundar en la afirmación de que lo que recoge esta

Tabla 8. Análisis confirmatorio muestra 2, segundo modelo.

\begin{tabular}{|c|c|c|c|c|c|c|c|c|c|c|c|}
\hline \multicolumn{6}{|l|}{ CMIN } & \multicolumn{6}{|l|}{ AIC } \\
\hline Model & NPAR & \multicolumn{2}{|c|}{ CMIN } & DF & $\begin{array}{l}\text { CMIN/ } \\
\text { DF }\end{array}$ & Model & AIC & \multicolumn{2}{|c|}{$\mathrm{BCC}$} & $\mathrm{BIC}$ & CAIC \\
\hline Default Model & 17 & \multicolumn{2}{|c|}{31,117} & .039 & 1,638 & Default Model & 65,117 & \multicolumn{2}{|c|}{73,170} & 96,927 & 113,927 \\
\hline Saturated Model & 36 & \multicolumn{2}{|c|}{.000} & 0 & & Saturated Model & 72,000 & \multicolumn{2}{|c|}{89,053} & 139,363 & 175,363 \\
\hline Independence Model & 8 & \multicolumn{2}{|c|}{238,453} & .000 & 8,516 & Independence Model & 254,453 & \multicolumn{2}{|c|}{258,243} & 269,423 & 277,423 \\
\hline \multicolumn{6}{|l|}{ RMR, GFI } & \multicolumn{6}{|l|}{ ECVI } \\
\hline Model & \multicolumn{2}{|c|}{ RMR } & \multirow{2}{*}{ GFI } & $\Delta G E I$ & DGES & Model & ECVI & \multicolumn{2}{|c|}{1090} & $H 190$ & MECVI \\
\hline Default Madel & \multirow{2}{*}{\multicolumn{2}{|c|}{19,866}} & & 767 & 463 & Default Model & 1,385 & \multicolumn{2}{|c|}{1,141} & 1,798 & 1,557 \\
\hline 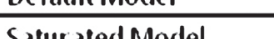 & & & 877 & , & . & Saturated Model & 1,532 & \multicolumn{2}{|c|}{1,532} & 1,532 & 1,895 \\
\hline \multirow{2}{*}{ Independence Model } & \multicolumn{2}{|c|}{, 000} & 1,000 & 153 & 266 & Independence Model & 5,414 & \multicolumn{2}{|c|}{4,444} & 6,543 & 5,495 \\
\hline & & & & & & \multicolumn{5}{|l|}{ HOELTER } & \\
\hline RMSEA & & & & & & Model & HOELTER.0 & & HOEL & TER.01 & \\
\hline Model & RMSE & & 1090 & $\mathrm{H} 190$ & PCLOSE & Default Model & 46 & & & 55 & \\
\hline & & & & & & Independence Model & 9 & & & 10 & \\
\hline Detault Model & ,116 & & ,026 & 188 & , 085 & Minimization: & 0 & 016 & & & \\
\hline Independence Model &, 400 & &, 354 & .447 & .000 & Miscellaneous: & & 250 & & & \\
\hline & & & & & & Bootstrap: & & 000 & & & \\
\hline & & & & & & Total: & & 266 & & & \\
\hline
\end{tabular}




\section{¿Es la Fibromialgia un trastorno depresivo con síntomas somáticos o es un síndrome de...}

escala, según nuestro punto de vista, no es la justificación de un trastorno mental como causa originaria de la FM, sino las consecuencias de un dolor real y mantenido en el tiempo que no es exclusivo de un sistema concreto sino de todo el organismo y que indica el estado general de agotamiento físico e incluso psicológico iniciado y/o mediado por la hiperactivación continua del eje HHA anteriormente citado. Es necesario en este punto tener en cuenta los datos de fiabilidad del test aportados por las escalas (IMN,IMP, INC e INF) que apoyan la veracidad de los síntomas detectados, quedando, por tanto, fuera de toda duda posibles exageraciones o manipulaciones por parte de los afectados.

La escala ANS revela unos niveles superiores a los considerados normales, (según el manual, menores a 59T). aunque las puntuaciones obtenidas están alejadas de problemas graves de ansiedad (91T o más) indicando que estos sujetos se encuentran en un estado intermedio de tensión y que no es la ansiedad la responsable principal de su deterioro generalizado de salud, sino más bien una consecuencia de otros problemas. Todas la subescalas se mantienen en medidas similares, aunque la ansiedad fisiológica (ANSF) marca de manera más significativa (72,50T). Esto puede ser indicador de que los niveles de ansiedad están más relacionados con las reacciones fisiológicas y no tanto con las cogniciones o las valoraciones emocionales, apoyando la idea de que es el malestar físico habitual el que tiene más peso en el estado de ansiedad de los pacientes con FM. Estos resultados coinciden con otros que señalan un perfil específico de afectados por la FM con mayores puntuaciones en las respuestas de ansiedad expresadas de forma fisiológica y motora ${ }^{35}$. Además, estos estados de tensión encontrados son coherentes con la hipervigilancia ante las señales de dolor y catastrofismo característicos de las personas afectadas por los $\mathrm{SSC}^{27}$.

Los valores alcanzados en la escala Depresión (DEP) por los sujetos experimentales son altos (media de 77T). Existe una evidente sensación de desesperanza y dificultad para tomar decisiones o para concentrarse. Principalmente los afectados acusan cansancio, bajos niveles de actividad, y una merma de energía y rendimiento físico debidos, entre otros, a las alteraciones en sus patrones de sueño y cambios en su apetito. Además - y muy probablemente como consecuencia de estos problemas- los niveles de tristeza son altos, hay una limitación en el interés por las actividades cotidianas y se observan dificultades para relajarse y disfrutar. Estos datos están en concordancia con investigaciones anteriores que recogen la prevalencia de la depresión en pacientes con SSC. Sin embargo, no se encuentran dentro de las puntuaciones consideradas como características de episodios de Depresión Mayor (81T o más), por lo que no podemos considerar que esta variable defina la depresión como una enfermedad en sí misma, sino una variable de personalidad relacionada con otras alteraciones encontradas en la enfermedad y siempre dentro de unos términos no psiquiátricos. En este sentido, nuestros datos refrendan estudios en los que marcan las diferencias entre la Depresión Mayor y los estilos cognitivos de afectados por FM. En concreto, algunos autores apuntan que, por ejemplo, las actitudes disfuncionales son menores en los afectados por FM que en los afectados por Depresión Mayor ${ }^{28}$. Otros encuentran también que dichas alteraciones en depresión no alcanzan cotas altas, y en los casos en que sí se alcanzan grandes cotas se debe sobre todo al tiempo transcurrido desde el diagnóstico ${ }^{36}$. Finalmente, ciertos investigadores entienden que, aunque el estado anímico alterado sea un nexo de unión para muchos de los afectados por FM y por otros SSC, el término depresión no refleja correctamente la realidad pues ni afecta por igual a todos los afectados ni puede considerarse un factor que explique por sí solo los SSC${ }^{7}$.

La escala Trastornos relacionados con la ansiedad (TRA) alcanza una puntuación significativa de $68,23 \mathrm{~T}$, la cual indica un grado de tensión habitual considerable y cierto grado de desconfianza o alerta en aspectos específicos de la vida. En cualquier caso, estos sujetos por lo general están muy alejados de problemas como la rumiación o trastornos generalizados de ansiedad (91T o más). Los niveles son absolutamente normales en trastorno obsesivo-compulsivo (TRAO) y en fobias (TRAF). En cambio, las puntuaciones en la subescala Estrés postraumático (TRAE) alcanzan niveles significativamente altos $(67,04 \mathrm{~T})$, lo cual puede estar relacionado con el hecho de que muchos pacientes aseguran haber vivido acontecimientos vitales muy estresantes y traumáticos a lo largo de la vida y en ocasiones relacionados con la aparición del dolor característico de la FM. Así lo demuestran algunos estudios que encuentran la acumulación de sucesos vitales estresante durante la niñez y adolescencia y lo relacionan con la existencia de $\mathrm{FM}^{37}$.

La escala Rasgos límites (LIM) nos presenta sujetos con niveles moderadamente elevados en cuanto a sensibilidad emocional, tendencia al cambio de humor y dudas acerca de aspectos vitales (LIME 65,75T y LIMI 66T), pero muy alejados de niveles patológicos graves como la impulsividad, la hostilidad o la ira (92T o más). En cambio las subescalas de relaciones interpersonales problemáticas (LIMP) y autolesiones (LIMA) puntúan bajas y dentro de la normalidad. Resulta lógico pensar que si la FM, al igual que otros SSC, están relacionados con la alteración del HHA y el mecanismo regulador del estrés, factores psicológicos como la labilidad emocional se vean afectados por esta activación permanente del Sistema Nervioso, tal y como afirma Eysenck en su teoría del Arousal/Activación ${ }^{38}$.

Los resultados obtenidos por los sujetos en la escala Esquizofrenia demuestran únicamente las dificultades que sufren estos a la hora de concentrarse o de mantener un control organizado de los procesos atencionales. Los estados de ansiedad, el dolor físico continuo, el ánimo bajo y el agotamiento habitual que hemos encontrado en otras escalas podrían justificar que las capacidades mentales, en muchos casos, se vean mermadas. De nuevo, el encontrar unas puntuaciones significativas en la subescala ESQA $(70,25 \mathrm{~T})$ pero muy alejadas de niveles de psicoticismo y esquizofrenia (90T o más) y que el resto de subescalas obtienen valores totalmente normales, nos hacen pensar que estos resultados sean la consecuencia de las otras características de la enfermedad y que esta escala por sí sola no pueda explicar una parte de la personalidad de los afectados.

Las puntuaciones obtenidas por el test CIF, como veíamos, indican un alto impacto de la enfermedad sobre la calidad de vida de los pacientes. El CIF recoge valores de capacidades motoras, valoración del bienestar, cansancio, estado de ánimo e intensidad de las molestias, entre otras. Por ello, se entiende que los altos valores obtenidos confirman los valores encontrados en las escalas del PAI.

En cuanto al cortisol, los niveles de las mujeres afectadas por FM en la primera muestra de nuestro experimento (46 mujeres) 


\section{LA. Díaz Robredo, et al.}

es de $6,842 \mathrm{nmol} / 1$ y son claramente más bajos que la media de mujeres sanas señaladas por otros estudios, como el de Aardal y $\mathrm{Holm}^{39}$, quienes encuentran niveles de cortisol matutino en mujeres libres de enfermedad de 51 a 60 años, con una media de $10.0 \mathrm{nmol} / 1$. Esto podría parecer estar en sintonía con algunos estudios que justifican la relación entre hipocortisolemia y FM. Sin embargo, otros estudios han evidenciado que afectadas de FM obtenían puntuaciones levemente superiores a mujeres sanas $^{18,19}$. Por otro lado, existen diferencias horarias entre las muestras tomadas en el experimento de Aardal y Holm ${ }^{38}$ el cual mide el cortisol a las 8:00 am y nuestro experimento que lo hace a las 10:30 am. Entendiendo que la hormona cortisol está sujeta a los ritmos circadianos y que existe una diferencia de más de dos horas entre ambos experimentos, la comparación entre sujetos sanos y afectados por FM es inviable. Por otro lado, el hecho de no haber limitado la inclusión de sujetos que toman de forma habitual diversas medicaciones que podrían afectar a los niveles basales de cortisol, hacen de nuevo que esta comparación sea difícil de determinar y de escasa generalización.

Los análisis factoriales exploratorios de la muestra 1 y 2 revelan la importancia de algunos de los factores de personalidad medidos por el PAI que hemos comentado (Somatización, Ansiedad, Depresión, Trastornos Relacionados con la Ansiedad, Paranoidismo, Esquizofrenia, Rasgos Límites e Impresión Negativa), del CIF y del cortisol a la hora de explicar la varianza común. Los análisis confirmatorios realizados posteriormente nos corroboran la relación existente entre los factores del PAI y la calidad de vida de los pacientes medida por el CIF en todos los casos. El cortisol no parece influir en ninguno de los supuestos. De los tres análisis confirmatorios realizados, es llamativo que el modelo formado por las escalas SOM, ANS y DEP ofrece una adecuación excelente y muy superior a los otros dos propuestos, expresando así la gran influencia de estas tres variables de personalidad en la calidad de vida de los pacientes.

Basándonos en estos resultados podemos concluir que la FM puede definirse como un SSC, caracterizado por unas variables de personalidad similares a las encontradas en otros SSC y explicadas principalmente por la alteración emocional, la ansiedad y las múltiples y persistentes quejas somáticas. Aunque el origen de la enfermedad no pueda demostrarse con los datos aportados por este experimento, sí podemos decir que los valores intermedios de las escalas sugieren que los rasgos de depresión, ansiedad y somatización no son el origen del problema ni estos pueden considerarse propios de personas aquejadas por alteraciones mentales como Depresión Mayor o Ansiedad Generalizada. Tal y como sugeríamos anteriormente, los valores de la escala Somatización no deben confundirse con otras enfermedades de origen psiquiátrico sino que resultan de la perpetuación en el tiempo de condiciones adversas de calidad de vida y dolor en los pacientes, por lo que el trato personal hacia estos pacientes, el diagnóstico y el tratamiento deberían tener en consideración la singularidad de los afectados. En cambio, la acumulación de factores traumáticos a lo largo de la vida y una sensibilidad física -mediada por el eje HHA- y psicológica -como la tendencia a la labilidad emocional- aumentadas ante los estímulos estresantes, podrían justificar dicha sintomatología y ser una vía de investigación futura hacia tratamientos que incrementen su calidad de vida.

\section{BIBLIOGRAFÍA}

1. Yunus, MB. Psychological aspects of syndrome: A component of the dysfunctional spectrum syndrome. Baillieres Clin Rheumatol.1994; 8: 811-837.

2. Weir PT, Harlan GA, Nkoy FL, Jones SS, Hegmann KT, Gren LH, Lyon JL. The incidence of fibromyalgia and its associated comorbidities: a populationbased retrospective cohort study based on International Classification of Diseases, 9th Revision codes. J Clin Rheumatol. 2006 Jun;12(3):124-8.

3. Yunus MB. Fibromyalgia and Overlapping Disorders: The Unifying Concept of Central Sensitivity Syndromes. Semin Arthritis Rheum. 2007 Jun; 36 (6): 339-356.

4. Bellato E, Marini E, Castoldi F, Barbasetti N, Mattei L, Bonasia DE, Blonna D. Fibromyalgia Syndrome: Etiology, Pathogenesis, Diagnosis, and Treatment. Pain Res Treat. 2012:426130.

5. Staud R. Abnormal endogenous pain modulation is a shared characteristic of many chronic pain conditions. Expert Rev Neurother. 2012 May;12(5):577-85.

6. Graven-Nielsen T, Arendt-Nielsen L. Peripheral and central sensitization in musculoskeletal pain disorders: an experimental approach. Curr Rheumatol Rep. 2002 Aug;4(4):313-21.

7. Yunus MB. Central Sensitivity Syndromes: A New Paradigm and Group Nosology for Fibromyalgia and Overlapping Conditions, and the Related Issue of Disease versus Illness. Semin Arthritis Rheum. 2008; 37: 339-352.

8. Salemi S, Rethage J, Wollina U, Michel BA, Gay RE, Gay S, Sprott H. Detection of interleukin 1 beta (IL-1beta), IL-6, and tumor necrosis factor-alpha in skin of patients with fibromyalgia. J Rheumatol. 2003;.30(1): 146-50.

9. Light KC, Bragdon EE, Grewen KM, Brownley KA, Girdler SS, Maixner W. Adrenergic dysregulation and pain with and without acute beta-blockade in women with fibromyalgia and temporomandibular disorder. J Pain. 2009 May;10(5):542-52.

10. Wood PB, Holman AJ. An elephant among us: the role of dopamine in the pathophysiology of fibromyalgia. J Rheumatol. 2009; 36(2): 221-224.

11. Carrasco GA, Van De Kar LD. Neuroendocrine pharmacology of stress. Eur J Pharmacol. 2003; 463(1-3): 235-272.

12. Myers B, Greenwood-Van Meerveld B. Role of anxiety in the pathophysiology of irritable bowel syndrome: importance of the amygdala. Front. Neurosci. 2009; 3: 47

13. Dinan T, Quigley E, Ahmed S, Scully P, O’Brien S, O’Mahony L, O'Mahony S, Shanahan F, Keeling PW. Hypothalamicpituitary- gut axis dysregulation in irritable bowel syndrome: plasma cytokines as a potential biomarker? Gastroenterology 2006; 130: 304-311.

14. Fukudo S, Nomura T, Hongo M. Impact of corticotropin-releasing hormone on gastrointestinal motility and adrenocorticotropic hormone in normal controls and patients with irritable bowel syndrome. Gut, 1998; 42(6): 845-849.

15. McCain GA, Tilbe KS. Diurnal hormone variation in fibromyalgia syndrome: a comparison with rheumatoid arthritis. J Rheumatol Suppl. 1989; 19: $154-7$.

16. Macedo JA, Hesse J, Turner JD, Meyer J, Hellhammer DH, Muller CP. Glucocorticoid sensitivity in fibromyalgia patients: decreased expression of corticosteroid receptors and glucocorticoid-induced leucine zipper. Psychoneuroendocrinology. 2008; 33(6): 799-809.

17. Izquierdo Álvarez S; Bancalero Flores JL, García Pérez MC, Serrano Ostariz E, Alegre de Miquel C, Bocos Terraz JP. Evaluación de la cortisoluria en mujeres diagnosticadas de fibromialgia. Med Clin (Barc), 2009; 133(7): 255-257.

18. Catley D, Kaell AT, Kirschbaum C, Stone AA. A naturalistic evaluation of cortisol secretion in persons with fibromyalgia and rheumatoid arthritis. Arthritis Care Res. 2000;13(1):51-61.

19. Díaz Robredo LA, Robles Sánchez JI. Estabilidad emocional y cortisol como factores diferenciadores de la fibromialgia. Sanid. mil. 2011; 67 (1): 11-17.

20. Fomicheva EE, Filatenkova TA, Rybakina EG. Activity of hypotnalamic-pituitary-adrenal axis by induction of experimental chronic fatigue syndrome.

21. Papadopoulos,AS, Cleare AJ. Hypothalamic-pituitary-adrenal axis dysfunction in chronic fatigue syndrome. Nat Rev Endocrinol. 2011 Sep 27;8(1):22-32.

22. Cuatrecasas G, Riudavets C, Güell MA, Nadal A. Growth hormone as concomitant treatment in severe fibromyalgia associated with low IGF-1 serum levels. A pilot study. BMC Musculoskeletal Disorders, 2007; 8: 119.

23. Loevinger BL, Shirtcliff EA, Muller D, Alonso C, Coe CL. Delineating Psychological and Biomedical Profiles in a Heterogeneous Fibromyalgia Population Using Cluster Analysis. Clin Rheumatol. 2012 Apr; 31(4):677-85. 


\section{¿Es la Fibromialgia un trastorno depresivo con síntomas somáticos o es un síndrome de...}

24. Waylonis, GW, Heck W. Fibromyalgia syndrome. New associations. Am J Phys Med Rehabil.1992;71: 343-348.

25. Giesecke T, Williams DA, Harris RE, Cupps TR, Tian X, Tian TX, Gracely RH, Clauw DJ. Subgrouping of fibromyalgia patients on the basis of pressure-pain thresholds and psychological factors. Arthritis Rheum. 2003 Oct;48(10):2916-22.

26. Henningsen P, Zimmermann T, Sattel H. Medically Unexplained Physical Symptoms, Anxiety, and Depression: A Meta-Analytic Review. Psychosom Med. 2003 Jul-Aug; 65(4):528-33.

27. Nijs J, Crombez G, Meeus M, Knoop H, Van Damme S, Van Cauwenbergh D, Bleijenberg G. Pain in Patients with Chronic Fatigue Syndrome: Time for Specific Pain Treatment? Pain Physician. 2012; 15:E677-E686.

28. Nordahl HM, Stiles TC. Personality styles in patients with fibromyalgia, major depression and healthy controls. Annals of General Psychiatry, 6:9.

29. Kroenke K, Spitzer R, Williams J. Physical symptoms in primary care: predictors of psychiatric disorders and functional impairment. Arch Fam Med. 1994 Sep;3(9):774-9.

30. Meeus M, Nijs J. Central sensitization: a biopsychosocial explanation for chronic widespread pain in patients with fibromyalgia and chronic fatigue syndrome. Clin Rheumatol (2007) 26:465-473.
31. Ford CV. Somatization and fashionable diagnoses: illness as a way of life. Scand J Work Environ Health. 1997;23 Suppl 3:7-16.

32. Mayou R, Kirmayer LJ, Simon G, Sharpe M. Somatoform disorders: time for a new approach in DSM V. Am J Psychiatry. 2005 May; 162(5):847-55.

33. Burckhardt CS, Clark SR, Bennett RM. The Fibromyalgia Impact Questionnaire: Development and validation. J Rheumatol. 1991 May;18(5):728-33.

34. Esteve-Vives J, Rivera J, Salvat MI, De Gracia M, Alegre C. Propuesta de una versión de consenso del Fibromyalgia Impact Questionnaire (FIQ) para la población española. Reumatol Clin. 2007; 3(1):21-4.

35. Pérez-Pareja J, Borrás C, Palmer A, Sesé A, Molina F, Gonzalvo J. Fibromialgia y emociones negativas. Psicothema, 2004; 16 (3): 415-420.

36. Máñez I, Fenollosa P, Martínez-Azucena A, Salazar A. Calidad del sueño, dolor y depresión en fibromialgia. Rev. Soc. Esp. Dolor 2005; 12: 491-500.

37. Imbierowicz K, Egle U. Childhood adversities in patients with fibromyalgia and somatoform pain disorder. Eur J Pain. 2003;7(2):113-9.

38. Eysenck HJ. The Biological basis of personality. Springfield, Il: Charles Thomas. 1967.

39. Aardal E, Holm AC. Cortisol in Saliva. Reference Ranges and Relation to Cortisol in Serum. Eur J Clin Chem Clin Biochem. 1995 Dec;33(12):927-32 